\title{
A petri net based reliability block diagram model for category I medical devices reliability analysis
}

\author{
L. Childs ${ }^{\mathrm{a}}$, K. Jenab ${ }^{b^{*}}$ and S. Moslehpour ${ }^{\mathrm{c}}$
}

a'Master's student at Morehead State University (150 University Blvd, Morehead, KY 40351 USA) in the Department of Engineering and Technology Management, USA

${ }^{b}$ Faculty of the Department of Engineering and Technology Management, Morehead State University, Morehead, KY 40351 USA ${ }^{c}$ Faculty of College of Engineering, Hartford University, 200 Bloomfield Avenue, West Hartford, CT 06117, USA

\section{H R O N I C L E \\ A B S T R A C T}

Article history:

Received: April 23, 2018

Received in revised format: June

24,2018

Accepted: August 9, 2018

Available online:

August 9, 2018

Keywords:

Medical equipment

Medical field

Petri Net

Reliability

Reliability block diagrams

Technology

\begin{abstract}
The medical industry incorporates technology and breaks different types of equipment into three various categories determined according to the technologies and their usage. The first category, which is the focus of this article, consists of devices that are directly linked to the life of the patients, for example a ventilator. The purpose of this study is to develop a new reliability technique, based on the Reliability Block Diagram (RBD) and Petri Net, for Category 1 equipment. The RBD, focuses on showing how the failure of different parts could affect the sub-systems of the equipment and how those failures could cause an overall system failure. The second method, Petri Net, is a tool that is used to analyze various types of information processing systems. Combining these two methods will allow the user to determine the reliability of the different systems and subsystems with various pieces of equipment. The knowledge gained by this analysis will be used to determine the likelihood that the failure of specific subsystems will cause an overall system failure. The overall anticipated result is to thoroughly develop this new methodology. The complete process that is used to finish the calculation process for the subsystems will be shown, in addition to the completion of the final Reliability Block Diagram.
\end{abstract}

\section{Introduction}

The use of medical devices can be traced throughout history, from the use of simple dental tools in Ancient Egypt to the modern-day pacemaker (Dhillon, 2011). The term medical equipment refers to a tool, for example a machine or instrument, that is used in the treatment, diagnosis, or prevention of illnesses or conditions, in addition to altering the structure of the body (Dhillon, 2000a). The number of tools and instruments used in the medical industry today is extremely wide and diverse. Due to that fact, equipment has been divided into three different categories based on the technology being used and its application (Dhillon, 2011). The first category of equipment, Category 1, contains devices that are directly linked to the life of the patient. Many different devices fall within this category, these include the pacemaker and the ventilator. The second category, Category 2, contains equipment that is primarily used for diagnostic purposes, such as X-Ray and Ultrasound machines. Lastly, equipment that falls under Category 3 is often referred to as convenience equipment, with an example being an electric hospital bed.

* Corresponding author.

E-mail address: k.jenab@moreheadstate.edu (K. Jenab)

(C) 2018 by the authors; licensee Growing Science, Canada doi: $10.5267 /$ j.msl.2018.8.008 
The hospitals and the medical industry today use over 5,000 different types of medical devices, with the reliability of these devices varying based on different factors, such as the purpose and complexity of the device (Dhillon, 2011). Reliability is defined as the probability that a tool will function at a designated standard under specific conditions for a designated period of time (Dhillon, 2000a). The different categories of equipment must meet various standards of reliability, with variation being contributed to how critical the operation of the machine is. The reliability of the first category of equipment must be extremely high and the machines must always be operational, because there is a little time for maintenance or repair. Many different techniques exist that can be used to determine the reliability of these devices. Some examples of these techniques include Failure Mode and Effects Analysis, Fault Tree Analysis and the Markov Method (Dhillon, 2000b). The overall purpose of this study is to develop a new method of analyzing the reliability of Category 1 medical equipment using a combination of the Petri Net technique and the Reliability Block Diagram (RBD).

The first technique that is going to be used in the creation of this new combination is the Petri Net (PN), which was developed in 1962 (Pastor et al., 1994). This method is both a graphical and mathematic system that is used to analyze and define information processing structures (Zaitsev, 2012). The graphical representation of this method is similar to that of flow charts and block diagrams and uses different types of nodes. A major factor that makes PNs different from the common diagrams previously mentioned, is the use of a token to simulate dynamic and concurrent events that will occur throughout the system (Pastor et al., 1994). The use of tokens in PNs are used to represent changes in states during the overall process, while the use of transitions in the diagram are what allow this representation to take place. The token and transition components of PNs are the main components of this method that are going to be used in the new combination technique. The second technique that is used in the creation of this new combination is the Reliability Block Diagram (RBD). The purpose of this type of diagram is to illustrate the logical and/or physical components of a system and depict the path of these factors, which are read from left to right. By analyzing the completed diagram, the total availability of the system can be calculated (Weyns et al., 2013). An advantage of using RBDs is that they can be used to analyze the different subsystems that make up a product or process. The analysis of subsystems and the detailed analysis of how an individual component can affect the overall system are the two main factors that lead to the selection of this method for the combination technique.

\section{Literature Review}

A greater understanding of four different subjects was needed in order to fully analyze and create the new methodology that this article proposes. First, the reliability of electronic equipment was investigated. The information that was uncovered showed different techniques that are used to analyze broad categories of devices, as well as standards that were created to ensure the needs of the customer and supplier were met (Amy \& Aglietti, 2009; Poncelin et al., 2010; Pechta et al., 2002). Once a basic understanding of equipment analysis was gained, then medical equipment reliability was specifically examined. The focus of this investigation was the different techniques that are currently in use. While investigating this topic it was discovered that some of the statistical methods that were being practiced, as well as some of the data that was being collected, was being done so incorrectly (Zaki et al., 2013). The next topic that was investigated was Petri Nets, specifically the different kinds that are available and different techniques that this method has been combined with in the past (Kounev et al., 2012; Vileiniskis et al., 2017; Ajmone Marsan et al., 1991). The last topic that was investigated was Reliability Block Diagrams including different combinations of techniques and how this technique has been used to study the reliability of electronics (Kim, 2011; Yanjun \& Wei, 2011; Weyns \& Höst, 2012; Weyns et al., 2013). The idea of combining different aspects of Petri nets with Reliability Block Diagrams is not a completely novel idea. Some limited documentation on the combined use of these techniques exists, however these approaches differ from the proposed idea in this research paper for several different reasons. One being that the versions of RBDs and PNs that are used in these techniques differ from the proposed approach, which uses aspects of the original concepts instead of altered versions. Some examples of the modified versions 
used in existing combinations include dynamic RBDs (Robidoux et al., 2010), colored PNs (Nebel et al., 2009), stochastic PNs (Ajmone Marsan et al., 1991). and PNs with aging tokens (Volovoi, 2004). Another difference is that many of these approaches have not been applied to a specific industry or field, while this approach is specifically designed and applied to conducting reliability analysis of Category 1 medical equipment. Lastly, very little published research has been conducted pertaining to the reliability analysis of Category 1 medical equipment and devices. The overall layout of this paper will include a description of the problem, specifically the need for this method of analysis and why a combination of these two reliability tools is needed. Also, a specific procedure pertaining to the use of this new combined technique will be created and thoroughly explained. Then, an illustrative example of this process will be presented and described. Lastly, a section showcasing the results of this combination and the future applications of it will be included.

\section{Problem statement}

The use of medical equipment has greatly increased over recent years, with the start of the increase dating back to the late 1990's, with the demand, complexity, and variety of items increasing. The manufacturing of medical equipment has become a major industry, with over 10,420 registered companies contributing to the production of these devices in 1997, with the number increasing due to the rise in demand of the products (Dhillon, 2000a). Due to the large manufacturing presence, the medical equipment industry also has a significant impact on the economy. The increased use of these devices has also led to other complications, such as the number of deaths directly caused by the use of faulty medical equipment. According to (Dhillon, 2011), a total of 1,200 deaths in the United States can be directly attributed to the use of defective equipment. The growth of the industry and the use of the equipment has also led to government regulations and oversight of all aspects of the product, including the design and manufacturing processes. The primary force of regulation in the United States for these standards is the Food and Drug Administration (Dhillon, 2000a). The factors and circumstances above help to showcase the need, not only for regulations for the design and production of these products, but also the need for reliability analysis and specific standards that should be met. Though reliability standards and checkpoints are needed for all medical equipment and devices, the need for sufficient reliability regulations and techniques for Category 1 equipment should be a priority. After an extensive research process, very few studies and articles were found specifically pertaining to the reliability of Category 1 equipment. Most medical equipment and technologies that are used today are referred to as complex systems, which means that the system is composed of a large quantity of components that locally and simultaneously interact (Poncelin et al., 2010). The many components and the complexity of the systems make calculating the reliability extremely difficult. A variety of different statistical techniques currently exist that can be used to examine and determine reliability, however some of these concepts are being applied incorrectly. A study was found that preformed an extensive investigation into the research that has been completed on the different techniques that are currently being used. The study primarily focused on medical equipment that measures continuous data, such as blood pressure, heart rate and oxygen levels. After finding over 500 articles, only 42 were included in this review and only four different methods were found. When they reviewed the articles, it was determined that eight of the articles showed inappropriate uses of statistical concepts, which could lead to the acceptance of faulty equipment. Based on the number of faulty techniques that were used, the conclusion was drawn that as many as two out of every ten medical devices that are currently in use could be faulty (Zaki et al., 2013). The process of determining the reliability of medical equipment is an extensive process, however finding an accurate way to determine this factor needs to be a priority. Also, due to the complexity of the different systems and the wide variety of products that are available, a broader range of statistical methods need to be investigated in order to meet the demand. A key objective when dealing with Category 1 medical equipment is that the reliability percentage must be greater, and the maintenance time must be extremely small. The new method involving Petri nets and RBDs that is being proposed, will provide a more extensive reliability analysis into the system, in addition to providing a tool to expedite the maintenance process and shorten equipment downtime. The RBD method was chosen for many different reasons, first it considers both the physical and logical 
components. Also, this method analyzes the subsystems and their relation to the overall system, specifically what failures can occur that will cause a complete system shutdown. The ability to allow the user to see the different relationships by using a graphical representation will help limit the amount of maintenance because the most likely causes and failures can be easily determined. The Petri Net model was selected because it can be used to analyze a variety of different systems and shows the different relationships and states of the various systems. Combining these methods will provide an analysis of the different subsystems and the states that the equipment can be in. The proposed methodology and step by step process of the method will be described in the next section of this paper.

\section{Methodology}

The proposed model of this paper is a Petri Net based Reliability Block Diagram, which includes significant aspects of both methods. The aspects of the RBD that will be included in this model are the relationships, analysis of subsystems, and reliability calculations for the components, including the subsystems, as well as the overall system. The relationships that will be used in the model are series, which are when all components are required for the system to operate, and parallel, where only one or a specified number of components have to be operational for the system to run. For example, a system could have two motors and the overall system will run if only one of the motors are operational. Also, it is important to note that if two different components are operating at the same time this is also a parallel relationship, but usually both elements must be operational for the system to function. The aspects of Petri nets that will be included in this diagram include transition bars, tokens and directional lines. The tokens are used to show at what point in the system the process is currently at, while the transition bars are used to show when the system is changing from one stage to another. Also, the directional lines are used to show the order of the processes, as well as which tokens must pass through the transition bar in order for the system to progress. Lastly, the equations that will be used to calculate the reliability of the system includes the reliability function for exponential distribution, the Mean Time to Failure (MTTF) calculation, and the series and parallel reliability equations. Overall, the model will provide an in depth look at the complete system, including the individual subsystems, and provide the total reliability of the system. A step by step procedure, including diagrams and equations, used to create this new model can be seen in the next section.

\subsection{Procedure}

The first step is to analyze the complete process and determine if any subsystems are included. Then, the relationships that makeup the overall system are examined, specifically the relationship between the subsystems and individual components. Next, a sequential layout of the subsystems and additional components is created.

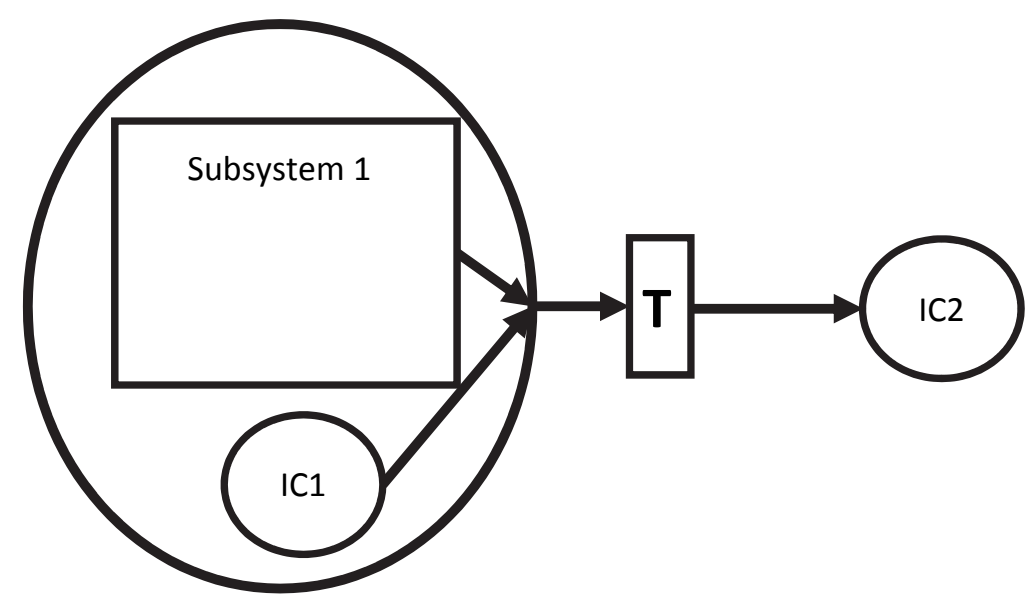

Fig. 1. Layout of an overall system constructed based on the instructions presented. 
For this diagram, large squares are used to represent the subsystems, while circles are used to represent the individual components. Once the order has been determined, transition blocks, which are represented by a small rectangle with a " $T$ " located in the center, are added between the different stages. Then, directional arrows are used to determine the flow of the network and which nodes connect to which transition point. Fig.1, depicts the layout of a system with both series and parallel relationships, which was constructed according to the steps previously presented. Once the basic layout is constructed, the next step is to analyze the different components that are included in each subsystem and determine if there are any parallel relationships included inside the subsystem. If any parallel relationships exist they will be grouped together using an oval shape, the reasoning behind this step will be explained later in the procedure. After the relationships are determined, the previous steps used to create the diagram for the overall system are used to produce the layout for the components located in the subsystem. The diagram for the subsystem will be placed inside the block that is designated for that specific subsystem. Then, the next step is to place the tokens at designated components in the diagram, which should be at the first component in each of the different "branches" that make up the process. Here is where the parallel relationships will be incorporated, if there is a parallel relationship at the start of a branch each of the different components will receive a token. The reasoning is that since the components have a parallel relationship, typically only one of them has to be operational in order for the system to function, so either one or both could pass the token to the transition block. When operations are occurring at the same time they both will receive a coin, however the next operation may not start until the transition bar receives coins from all the operations. Lastly, once the token located in the subsystem has reached the last component, it will exit the subsystem and go to the corresponding transition block. Tokens are also placed at the first activity of a branch in the overall network diagram as well. A diagram that shows the layout of the complete system with the finished subsystems and tokens can be seen below in Fig. 2.

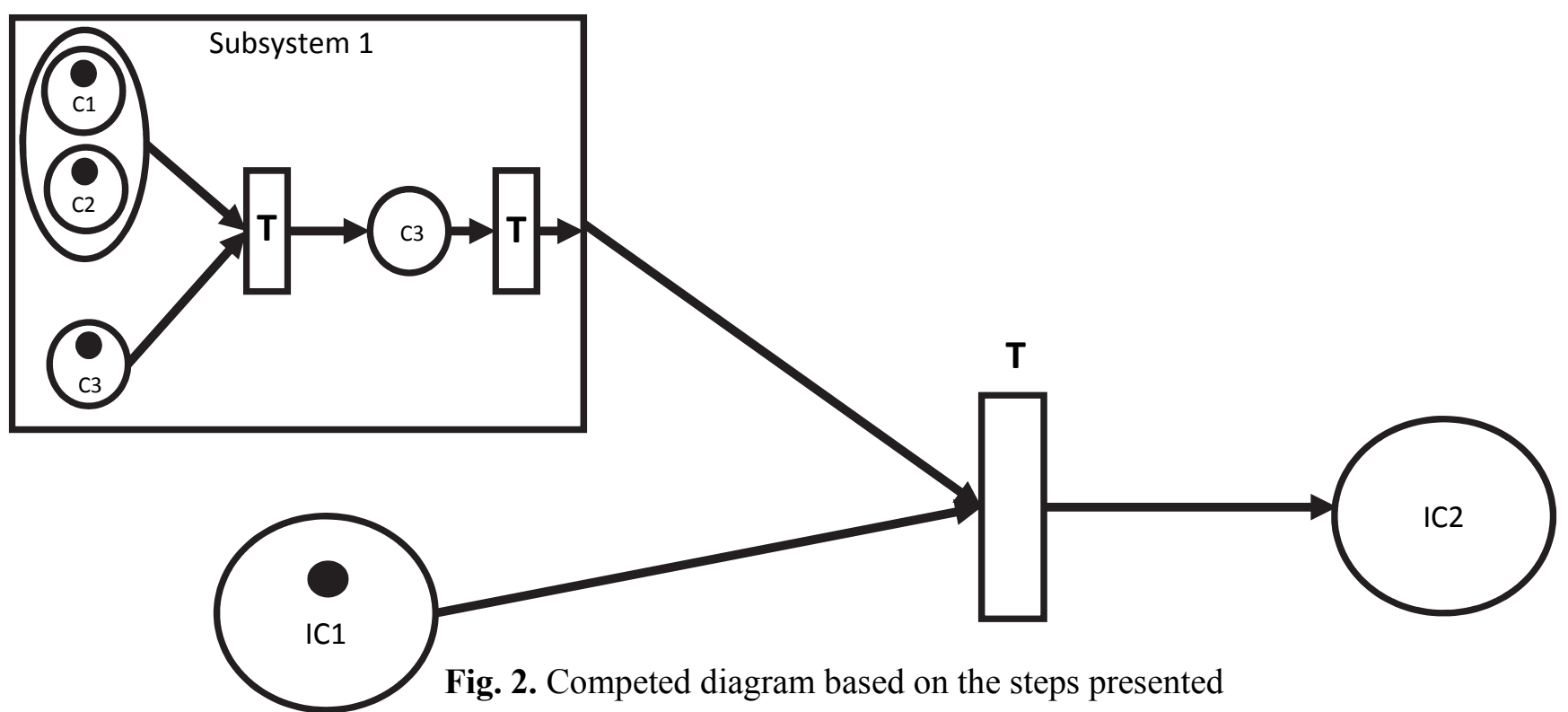

The last step to complete the model is to calculate the reliability of the overall system. To complete this process the first step is to calculate the reliability of each component using the equations for reliability function for exponential distribution (1). The factors used to determine this value is $\lambda$, which is the inverse of the Mean Time to Failure Value, and the time, $t$, over which the product was investigated.

$$
\text { Relaibility }=e^{-\lambda t} .
$$

The next expression, Eq. (2), was used to calculate the MTTF. Using the variables $t$, which is the amount of time that the products were examined, and $n$, which is the number of products that were tested.

$$
\text { MTTF }=\frac{t}{n} \text {. }
$$


Once those values have been calculated the reliability of each component is added to their specific symbol located in the previously completed model. Then, the parallel and series equations seen below are used to calculate the reliability of the subsystems first, then the overall system. For the parallel equations, if all the components in the parallel structure must be completed in order for the system to function then the individual reliability values are multiplied together. The Eq. (3), is used to calculate the parallel reliability of the system, where $R$ is the individual reliability of the components.

$$
\text { Parallel Reliability }=1-\left(1-R_{1}\right) \times\left(1-R_{2}\right) \times \ldots \times\left(1-R_{n}\right)
$$

The series reliability, Eq. (4), is also calculated using the individual reliability of each component that makes up the product.

$$
\text { Series Reliability }=R_{1} \times R_{2} \times R_{3} \times \ldots \ldots \times R_{n} .
$$

When calculating the systems, an expression will be constructed based on the created diagram that will combine the series and parallel equations based on determined relationships. Lastly, the final calculated value will be compared to the company's required reliability for that piece of Category 1 equipment to determine if the device meets the required standard.

\section{Illustrative example}

An illustrative example of this unique process can be seen in this section, where a hypothetical piece of Category 1 medical equipment is analyzed. The components, the direct predecessors for each one, and the relationship between the components for the overall system can be seen in Table 1.

\section{Table 1}

\begin{tabular}{|c|c|c|c|}
\hline Component & Predecessor & Relationship & \\
\hline Subsystem $1\left(\mathbf{S}_{1}\right)$ & $\begin{array}{ll}--- \\
-\end{array}$ & \multirow{2}{*}{ Parallel (One of Two Operational) } & \\
\hline Subsystem $2\left(\mathbf{S}_{2}\right)$ & ---- & & \\
\hline $\mathbf{P}_{1}$ & $\mathrm{~S}_{1}, \mathrm{~S}_{2}$ & \multirow{2}{*}{ Parallel (Both Operational) } & \\
\hline Subsystem $3\left(\mathbf{S}_{3}\right)$ & $\mathrm{S}_{1}, \mathrm{~S}_{2}$ & & Series \\
\hline $\mathbf{P}_{2}$ & $\mathrm{P}_{1}, \mathrm{~S}_{3}$ & \multirow{3}{*}{$\begin{array}{c}\text { Parallel (Both Operational) } \\
\text { Series }\end{array}$} & \\
\hline $\mathbf{P}_{3}$ & $\mathrm{P}_{1}, \mathrm{~S}_{3}$ & & \\
\hline $\mathbf{P}_{4}$ & $\mathrm{P}_{2}, \mathrm{P}_{3}$ & & \\
\hline
\end{tabular}

The direct predecessors for each one, and the relationship between the components

Based on the data presented above, the overall system layout is constructed, where the transitions are represented by a rectangle with a " $\mathrm{T}$ " located in the center, the subsystems are represented by a square and the individual components are represented by a circle. The parallel relationships in this system are contained within an oval, as directed by the instructions in the previous section. The next step is to analyze and construct the three different subsystems. The second table, Table 2, shows the relationship type, predecessors, and components for each of the three subsystems.

\section{Table 2}

\begin{tabular}{|c|c|c|}
\hline \multicolumn{3}{|c|}{ Subsystem 1} \\
\hline Component & Predecessor & Relationship \\
\hline $\mathbf{A}_{1}$ & ---- & Parallel (One of Two Operational) \\
\hline $\mathbf{A}_{2}$ & ---- & \\
\hline $\mathbf{A}_{3}$ & $A_{1}, A_{2}$ & Series \\
\hline $\mathbf{A}_{4}$ & $\mathrm{~A}_{3}$ & Series \\
\hline \multicolumn{3}{|c|}{ Subsystem 2} \\
\hline Component & Predecessor & Relationship \\
\hline $\mathbf{B}_{1}$ & ---- & Parallel (One of Two Operational) \\
\hline $\mathbf{B}_{2}$ & ---- & \\
\hline $\mathbf{B}_{3}$ & $\mathrm{~B}_{1}, \mathrm{~B}_{2}$ & Series \\
\hline $\mathbf{B}_{4}$ & $\mathrm{~B}_{4}$ & Series \\
\hline \multicolumn{3}{|c|}{ Subsystem 3} \\
\hline Component & Predecessor & Relationship \\
\hline $\mathrm{C}_{1}$ & --- & Parallel (One of Two Operational) \\
\hline $\mathrm{C}_{2}$ & ---- & \\
\hline $\mathrm{C}_{3}$ & $\mathrm{C}_{1}, \mathrm{C}_{2}$ & Series \\
\hline
\end{tabular}

The relationship type, predecessors, and components for each of the three subsystems 
After examining the relationships, the layout for the three different subsystems is created and then placed in the designated location on the overall system model. Also, the tokens are added to the first activity in a "branch" during this step as well. The complete overall system model can be seen below in Fig. 3 .

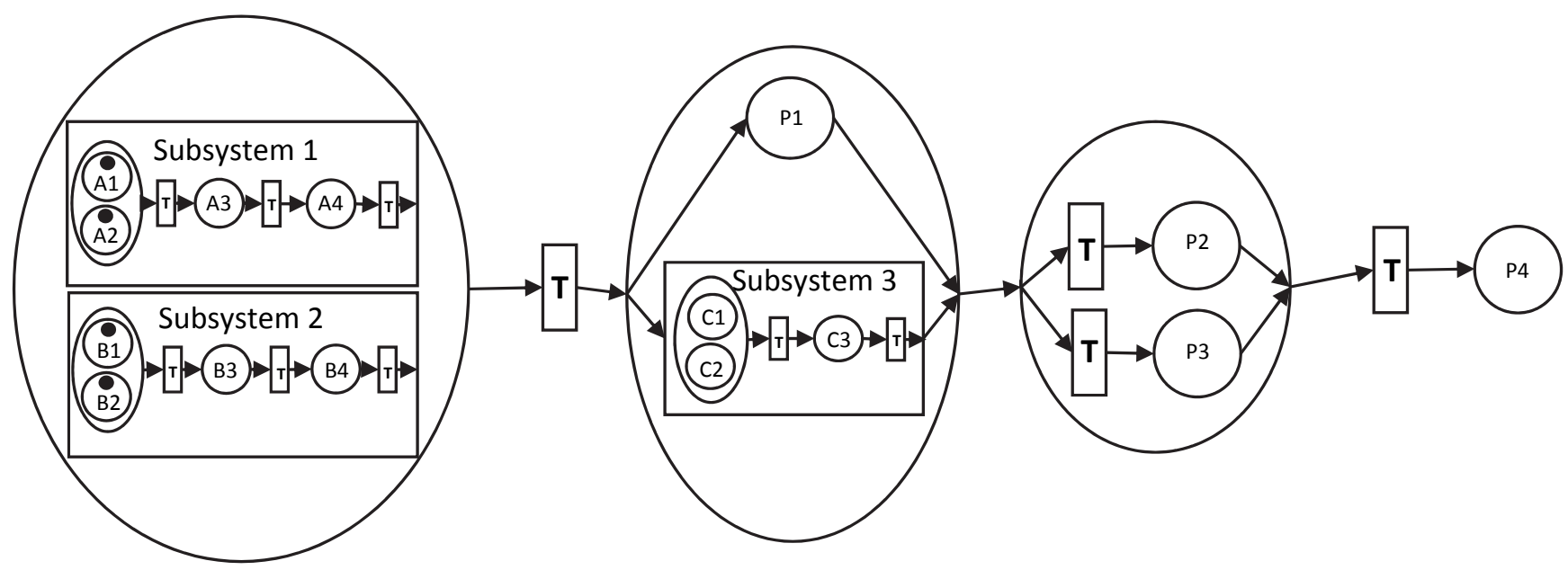

Fig. 3. Complete system model with tokens.

The next step is to calculate the reliability of each of the different components, given the Mean Time to Failure (MTTF) values provided by the company that produces the product. Then, Eq. (1) is used to calculate the reliability of each of the components, an example of the calculation process used to analyze component $\mathrm{P}_{1}$ can be seen below. This process, Eq. (5), is then duplicated for each of the different components, even the components in the subsystems.

$$
\text { Reliability }_{\boldsymbol{P}_{\mathbf{1}}}=e^{-\left(\frac{1}{M T F F}\right) t}=e^{-\left(\left(\frac{1}{1500}\right) 150\right)}=\mathbf{0 . 9 0 5}
$$

The table shown below, Table 3, displays the given MTTF values and the calculated values of reliability for each component.

\section{Table 3}

MTTF values and the calculated values of reliability for each component

\begin{tabular}{|c|c|c|c|}
\hline Component & Meant Time to Failure (MTTF) & Time (t) & Reliability \\
\hline $\mathbf{P}_{1}$ & 1500 hours & 150 hours & 0.905 \\
\hline $\mathbf{P}_{2}$ & 17000 hours & 200 hours & 0.988 \\
\hline $\mathbf{P}_{3}$ & 2000 hours & 210 hours & 0.900 \\
\hline $\mathbf{P}_{4}$ & 1700 hours & 175 hours & 0.902 \\
\hline \multicolumn{4}{|c|}{ Subsystem 1} \\
\hline $\mathbf{A}_{1}$ & 1750 hours & 200hours & 0.892 \\
\hline $\mathbf{A}_{2}$ & 1500 hours & 150 hours & 0.905 \\
\hline $\mathbf{A}_{3}$ & 2000 hours & 225 hours & 0.894 \\
\hline A4 & 1700 hours & 175 hours & 0.902 \\
\hline \multicolumn{4}{|c|}{ Subsystem 2} \\
\hline $\mathbf{B}_{1}$ & 1750 hours & 200 hours & 0.892 \\
\hline $\mathbf{B}_{2}$ & 1500 & 150 hours & 0.905 \\
\hline $\mathbf{B}_{3}$ & 2000 & 225 hours & 0.894 \\
\hline $\mathbf{B}_{4}$ & 1700 & 175 hours & 0.902 \\
\hline \multicolumn{4}{|c|}{ Subsystem 3} \\
\hline $\mathbf{C}_{1}$ & 1500 & 150 hours & 0.905 \\
\hline $\mathbf{C}_{2}$ & 1700 & 200 hours & 0.889 \\
\hline $\mathrm{C}_{3}$ & 2000 & 200 hours & 0.905 \\
\hline
\end{tabular}


After the reliability of each component is calculated, each of the subsystems are analyzed individually and the overall reliability of each is determined using the formulas for series given in Eq. (4) and parallel reliability in Eq. (3). The calculation process for each of the subsystems can be seen in Table 4.

Table 4

The Calculation process for each of the subsystems

\begin{tabular}{|c|}
\hline Subsystem 1 \\
\hline Parallel $_{1}=A_{1} \| A_{2}=1-\left(1-A_{1}\right) \times\left(1-A_{2}\right)=1-(1-0.892) \times(1-0.905)=0.9897$ \\
\hline Subsystem $_{1}=\left(\right.$ Parallel $\left._{1}\right) \times A_{3} \times A_{4}=(0.905)(0.894)(0.902)=0.73$ \\
\hline Subsystem 2 \\
\hline Parallel $_{2}=B_{1} \| B_{2}=1-\left(1-B_{1}\right) \times\left(1-B_{2}\right)=1-(1-0.892)-(1-0.905)=0.9897$ \\
\hline Subsystem $_{2}=\left(\right.$ Parallel $\left._{2}\right) \times B_{3} \times B_{4}=(0.905)(0.894)(0.902)=0.73$ \\
\hline Subsystem 3 \\
\hline Parallel $_{3}=C_{1} \| C_{2}=1-\left(1-C_{1}\right) \times\left(1-C_{2}\right)=1-(1-0.905) \times(1-0.889)=0.9895$ \\
\hline Subsystem $_{3}=\left(\right.$ Parallel $\left._{3}\right) \times C_{3}=0.9895 \times 0.905=0.8955$ \\
\hline
\end{tabular}

The calculated reliability values are then added to the diagram that was previously generated and the complete diagram can be seen below in Fig. 4.

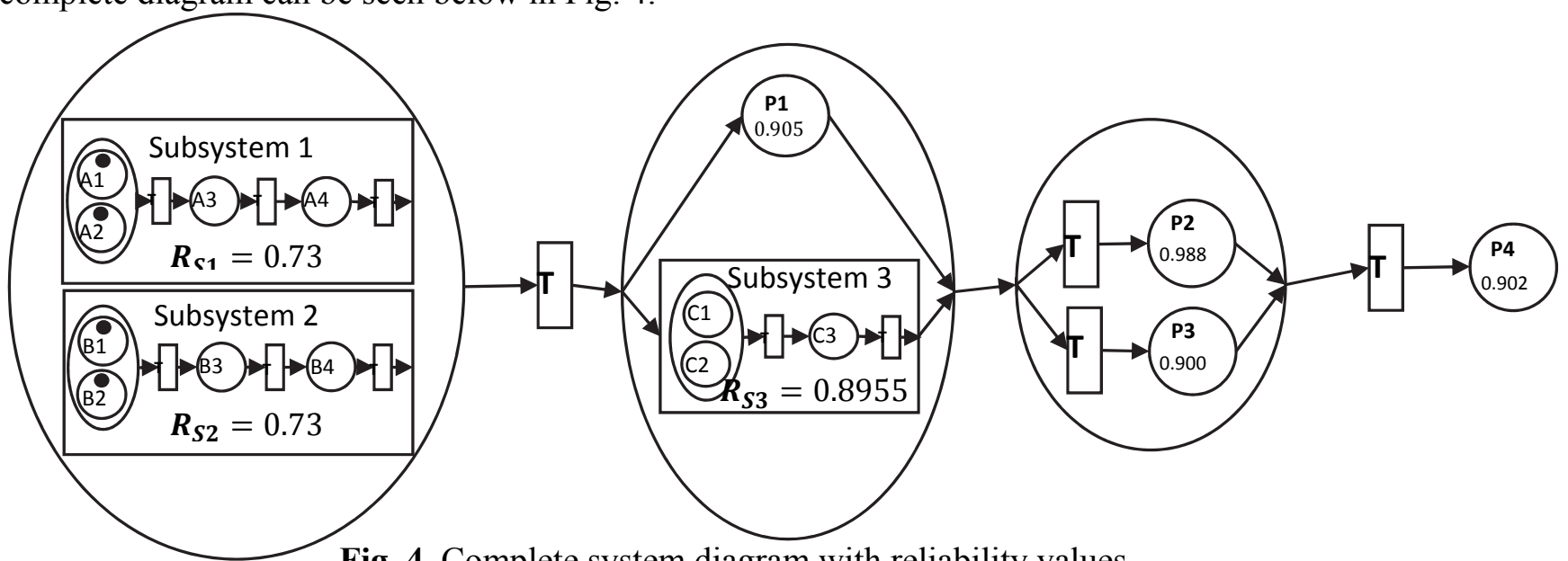

Fig. 4. Complete system diagram with reliability values

Once the subsystems have been calculated, the overall system reliability is determined using the same equations as the process above. Two of the parallel relationships that are seen in the overall structure specify that all the activities in those relationships must be completed so the reliability will be multiplied. The complete calculation process can be seen below in Table 5 .

\section{Table 5}

The results of the computations

\begin{tabular}{|c|c|}
\hline Parrallel $_{4}$ & Parallel $_{6}$ \\
\hline $\begin{array}{c}R_{S 1} \| R_{S 2}=1-\times\left(1-R_{s 2}\right)=1-(1-0.73) \times(1-0.73)=0.9271 \\
R_{P 1} \| R_{S 3}=0.905 \times 0.8955=0.8104\end{array}$ & $R_{P 2} \| R_{P 3}=0.988 \times 0.900=0.8890$ \\
\hline $\begin{array}{c}\text { Complete System } \\
\text { Parallel }_{4} \times \text { Parallel }_{5} \times \text { Parallel }_{6} \times P_{4}=0.9271 \times 0.8104\end{array}$ & $8890 \times 0.902=0.6025$ \\
\hline
\end{tabular}

The overall calculated reliability of the system is then compared with the designated standard to determine if the piece of equipment is acceptable. By completing the calculation process for the reliability of the entire system, it is determined that the complete system reliability is 0.6025 . Analyzing the complete diagram shows that the combination of Subsystem 3 and $\mathrm{P}_{1}$ have the smallest reliability value, which would be where the most problems are likely to occur. 


\section{Conclusion}

The demand and variety of medical equipment that are available today have led to the creation of three different categories of medical equipment, with the lives of patients directly relying on Category 1 devices. A minimal amount of research exists today pertaining to the reliability of Category 1 equipment. The lack of research pertaining to this topic, as well as an increasing need for techniques to analyze these devices, led to the development of this method. The technique consisted of combining two analysis methods, Petri Nets and RBDs, to examine the reliability of components and subsystems that make up Category 1 equipment. The use of this technique has led to the creation of a new type of reliability diagram that considers the reliability of each component and subsystem. By following the procedure presented in this article, an individual will be able to analyze any given piece of equipment that falls within this category. The technique that was developed would allow the user to obtain detailed information about many different aspects of the product. First, the diagram will allow the individuals to see the relationships between all of the components in the overall system. The relationship of the different subsystems within the overall system, such as whether they work in series or parallel, can be seen in this diagram. Also, by using the token system that is one of the major aspects of Petri Nets, the user will be able to see the flow of the process and determine where the error occurred. In addition, the reliability of each component is included in the diagram making it easier for an individual to determine which components to examine first when a failure occurs. Lastly, this system has the user analyze each subsystem individually before including them in the overall diagram. By analyzing the different subsystems, a greater understanding of the layout, as well as where possible errors are most likely to occur can be determined. While completing the illustrated example, some of the limitations of this technique became clear. One of the limitations is that the diagram can get very confusing when there are many different components and subsystems involved. To rectify this problem the user could break the diagram up into sections and create a simplified diagram of the overall process. Another factor is that the user who creates the diagram must either be an expert on the equipment or have detailed information about the machine from the company about the components and subsystems, specifically the relationship and layout. Overall, this new method that was developed will provide individuals and companies with the tools needed to increase the overall reliability of these products. The new technique will also give technicians a better idea of where to start the trouble shooting and maintenance process. Further work needs to be done to determine the overall impact and usefulness of this new technique. Also, a real-world example that analyzes a machine that is currently on the market should be completed.

\section{References}

Ajmone Marsan, M., Balbo, G., Chiola, G., Conte, G., Donatelli, S., \& Franceschinis, G. (1991). An Introduction to Generalized Stochastic $\{\mathrm{P}\}$ etri Nets. Microelectronics and Reliability.

Amy, R., \& Aglietti, G. (2009). Reliability analysis of electronic equipment subjected to shock and vibration - A review. Shock and Vibration, 16, 45-59. https://doi.org/10.3233/SAV-2009-0453

Dhillon, B. S. (2000a). Chapter 1: Introduction. In Medical Device Relability and Associated Areas (pp. $1-9)$.

Dhillon, B. S. (2000b). Chapter 3: Tools for Medical Device Reliability Assurance. In Medical Device Relability and Associated Areas (pp. 31-50).

Dhillon, B. S. (2011). Medical Equipment Reliability: a Review, Analysis Methods and Improvement Strategies. International Journal of Reliability, Quality and Safety Engineering, 18(04), 391-403.

Kim, M. C. (2011). Reliability block diagram with general gates and its application to system reliability analysis. Annals of Nuclear Energy, 38(11), 2456-2461.

Kounev, S., Spinner, S., \& Meier, P. (2012). Introduction to queueing petri nets. Proceedings of the Third Joint WOSP/SIPEW International Conference on Performance Engineering - ICPE '12, (August 2014), 9. https://doi.org/10.1145/2188286.2188290

Nebel, S., Dieter, A., \& Bertsche, B. (2009). Application of extended coloured stochastic petri nets for modelling and analysis of RAMS $+\mathrm{C}$ of production systems. IFAC Proceedings Volumes (IFACPapersOnline), 2(PART 1), 163-168. https://doi.org/10.3182/20090610-3-IT-4004.00033 
Pastor, E., Roig, O., Cortadella, J., \& Badia, R. M. (1994). Petri net analysis using boolean manipulation, 416-435. https://doi.org/10.1007/3-540-58152-9_23

Pechta, M., Dasa, D., \& Ramakrishnanb, A. (2002). The IEEE standards on reliability program and reliability prediction methods for electronic equipment. Microelectronics Reliability, 42(9), 12591266. https://doi.org/10.1016/S0026-2714(02)00132-4

Poncelin, G., Cauvin, A., Glade, M., Lyonnet, P., \& Dufrene, D. (2010). Design for electronic equipment reliability in complex systems. International Journal of Product Development.

Robidoux, R., Xu, H., Member, S., Xing, L., \& Member, S. (2010). Automated Modeling of Dynamic Reliability Block Diagrams Using Colored Petri Nets, 40(2), 337-351.

Vileiniskis, M., \& Remenyte-Prescott, R. (2017). Quantitative risk prognostics framework based on Petri Net and Bow-Tie models. Reliability Engineering and System Safety, 165(September 2016), 62-73.

Volovoi, V. (2004). Modeling of system reliability Petri nets with aging tokens.pdf.

Weyns, K., \& Höst, M. (2012). Risk Analysis for Critical Systems with Reliability Block Diagrams. Iscramlive.Org, (April), 1-5. Retrieved from http://www.iscramlive.org/ISCRAM2012/proceedings/239.pdf

Weyns, K., Weyns, K., \& Höst, M. (2013). Case Study on Risk Analysis for Critical Systems with Reliability Block Diagrams, (May), 693-702.

Yanjun, L., \& Wei, W. (2011). Research on the System of Relibaility Block Diagram Design and Reliability Prediction. International Conference on System Science, Engineering Design, Manufacturing Informization, 35-38.

Zaitsev, D. A. (2012). Universal petri net. Cybernetics and Systems Analysis, 48(4), 498-511.

Zaki, R., Bulgiba, A., Nordin, N., \& Ismail, N. A. (2013). A systematic review of statistical methods used to test for reliability of medical instruments measuring continuous variables. Iranian Journal of Basic Medical Sciences, 16(6), 803-807. https://doi.org/10.1371/journal.pone.0037908

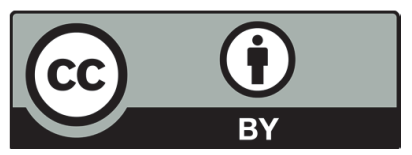

(C) 2018 by the authors; licensee Growing Science, Canada. This is an open access article distributed under the terms and conditions of the Creative Commons Attribution (CCBY) license (http://creativecommons.org/licenses/by/4.0/). 